\title{
58
}

\section{E-discourse in education}

\author{
Jan Perkins
}

McMaster University

Ontario, Canada

Kenneth Newman

North Lakehead University

Ontario, Canada

\begin{abstract}
This paper is about production, transmission and consumption of electronic text on computers as a new and distinct form of discourse. It argues that understanding styles and social relationships found in this e-discourse is useful for learning and practice, and for achieving communicative competence. Various media which employ e-discourse are discussed, emphasizing their role in articulating new personal and professional relationships in new ways. Several prevailing notions about e-discourse, including asynchronicity, egalitarianism, absence of social cues and candour are critically examined and revised. A cross-section of literature on experiences with Computer Mediated Communication (CMC) is synthesized with the authors' experiences in using and teaching e-discourse, paying equal attention to factors in failure as well as success.
\end{abstract}

Main conference themes: integration

Educational areas: higher education

Study topics:

Secondary keywords: communication, curriculum development, electronic mail, telecommunications, thinking 


\section{INTRODUCTION}

The past fifteen years have seen rapid development in production and consumption of electronic text for telecommunication by computers. Parallel to this growth, scholars have become aware of peculiarities of communicative style in these media. We propose that these characteristics of exchanging electronic text are so profound and particular that computer mediated communication (CMC - a term we would employ except that we are strictly concerned with electronic text, which we call e-text) must be considered distinct from other forms of expression. Furthermore, already exhibiting a robust 'life of its own', it should be considered as a separate mode of discourse susceptible to and able to benefit from the same levels of analysis and understanding as any other mode. We propose the term 'e-discourse' for the totality of communication employing e-text transmitted over computer networks, including its social context.

E-discourse offers participants tremendous expressive potential and several distinct hazards. Due to its increasing presence in our personal and professional lives, especially in education, we argue that e-discourse can and should be understood. High levels of competence can be achieved by experts engaged in practice, whom we call 'virtuosos'. Their skills can be learned through observation and analysis. These can and should be learned by anyone who needs to use e-discourse.

\section{CHARACTERISTICS OF E-DISCOURSE}

E-discourse, though still nascent, has been with us long enough that, despite its appearance as text, many have recognized that there is something very different about it. E-discourse is no less than a new form of both writing and reading. Writing in its new electronic form can substitute for speech, in many senses doing what speech can do and more.

E-discourse is also a way of mediation for new forms of social relationship. This mode of discourse encompasses a number of paradoxes and ambiguities which can be used to great advantage in these new relationships. Harasim [1, p. 25] has noted, "text based messaging creates a new form of interpersonal interaction with advantages over postal mail, telephone calls and even face-toface encounters. It is at once direct while informal ...".

Part of what makes e-mail messages different from letters is that they have no physical presence. One does not save and cherish them. One rarely stares at them in dread. And we don't erase real letters the way we send e-mail to the 
bit bucket. These features cause us to consider its nonartefactual nature an essential element of e-discourse, at least for the present.

This apparent ephemerality of e-text has advantages and disadvantages. It removes a barrier to communication. One can easily dash off a message. But in this very speed and convenience, especially in comparison to the artefacts one sends by regular post, there is the risk that a sender might not devote enough thought to a communicative utterance which should require care and artifice. Correspondingly, a recipient might not give an e-mail the scrutiny or weight it deserves. This potential pitfall contrasts with printed material and the risk of taking physical text too seriously simply because it is print.

\section{Metacommunciation}

Metacommunication is communication about communication. It may be as simple as a comment about delays in message transmission or as complex as Wittgenstein's linguistic philosophy. Feenberg [2, p. 34] has pointed out the tendency to metacommunication in e-discourse, noting how purposeful and necessary it is to the various media by which it is channelled. One of the most frequently remarked phenomena in e-discourse is flaming, the frank expression of extreme argumentativeness up to and including actual verbal abuse. Kiesler, Seigel and McGuire [3] comment on a similar phenomenon seen in the earliest forays into e-discourse with the teletype machine. But this need not be entirely negative. As Boshier [4, p. 57] remarked, with more ambivalence than most, "flaming is a stimulating feature of e-mail".

Flaming may well be just one expression of a paradox of candour. Ediscourse also leads some users to dishonesty without concern for responsibility and others to an openness without concern for risks. Dishonesty can include the usual scams such as chain letters and get-rich schemes, specious claims to authority and, as grippingly detailed by Van Gelder [5] in "The Strange Case of the Electronic Lover", emotional and intellectual fraud through an ostensibly playful adoption of personae and alter egos. Openness can include such risky behaviours as making embarrassing personal admissions on conferences, writing too familiarly to those with greater power who may be resentful, or indulging in caustic, blazing discussions ultimately as embarrassing to participants as to innocent bystanders. In situations where self disclosure or display of strong emotions can entail repercussions, posters should be aware that the seductive informality of e-discourse carries risks. This is especially pertinent to users in educational institutions and in other work environments. The very feature which leads many to champion electronic communication, its apparent openness, can later cause considerable problems. Educators ought to prepare themselves and their students for these effects. 


\section{The element of time}

Handling the element of time in e-discourse is more complex then it seemed at first, when much was made of asynchronous messaging. E-discourse does not lack a temporal dimension. Exchange of messages or utterances proceeds in forward, relatively linear fashion. This is so despite lateral branching by topic. Furthermore, in most communicative exchanges there is a flow or cadence, usually arrived at among parties as a consensual understanding, though it could as easily be the product of concerted metacommunication. Skilful communicators learn nuances of time particular to e-discourse and are able to use them for greater efficiency. For example, virtuosos usually compose and edit messages off-line, quote previous messages or substantially edited exchanges to provide context and provide occasional summaries.

\section{Social relationships}

The variety of social relationship possible with e-discourse is perhaps the element arguing most strongly its distinctness. Again it evokes paradox, this time one of individuality and collectivity. Messages may be interpreted both as an expression of the individuality of a contributor and of a collectivity, such as a list or conference within a community of shared interest. Despite its technological channels, this communication remains a very human and social activity, with its shared history, in jokes and other forms of association or affinity [6]. As Kiesler [7, p. 159] stated in the case of distributed work groups, "these are fundamentally new kinds of social organisation, not anticipated or explained by existing social theory". On a list there is both a sense of personal interaction between the two individuals who respond to each other in a thread and of the collective articulation of thought shared with other list members, including those who participate by lurking.

We see a social shift, recreationally and especially occupationally, as ediscourse collectivities develop on an increasingly lateral frame across areas of shared interest, bypassing traditional hierarchies though leaving them intact. For example, a LAN manager in a medium sized corporation might turn to a counterpart at another organisation for help before consulting an in house colleague or superior. An educator preparing a class on sexual harassment might contact through e-mail a colleague at another university rather than their own department.

In our experience with physiotherapy students on a BBS system, we also observed the tendency to preferentially develop lateral relationships. Attempts by faculty to start clinical discussions failed. Yet when students were separated from their peers and actually treating clients, they began to generate clinical discussion among themselves, publicly and privately. A colleague observed a similar phenomenon among students in a family medicine programme who used 
the same messaging system. The artificial clinical discussions started by educators, in which participation was voluntary, were a failure. Much later, similar discussions rooted in actual need were observed to develop spontaneously among the students.

\section{COMMON MYTHS AND MISUNDERSTANDINGS}

\section{E-discourse has a democratizing effect}

When people began to use e-discourse, particularly within groups, early observations fuelled speculation that the medium itself was inherently egalitarian, that it had a democratizing effect. In education these ideas often came out of case studies, particularly those done within programmes designed to foster cooperation and high levels of interactivity. However, in different settings others have not noticed the same effect. Kiesler [7, p. 159], who has looked at the introduction of information technology in a variety of settings, noted that the revolutionary changes predicted by some have not occurred, "Before we speculate too much, the evidence to date has not demonstrated the withering of hierarchy in economic organization, much less in educational organization". What she describes is an amplification effect. Strongly centralized organizations which introduced networks, used the technology in ways which reinforced centralization, while decentralized organizations moved towards further decentralization. In other words, style and structure of an organization are the determinant factors.

In early work in education, observers noticed that even novice users were determined to work around the lack of contextual information and to restore a status system. Students at the Open University using CoSy responded to the lack of cues by such methods as searching out resumes of message posters, or by using the information deduced from messages to rebuild a status system [8].

\section{Absence of social cues}

A related notion about e-discourse, despite conflicting evidence, is the absence of social cues. There is a flattening or loss of the social cues particular to face to face communication, which may help explain some of the informality and the readiness with which people engage in aggression and self disclosure. But there remain plenty of other social cues, particularly for a virtuoso. These go well beyond such obvious stratagems as emoticons and acronyms.

Among strangers in electronic discussion, people are represented only by their communicative competence. Claude Baltz, quoted by Feenberg [2, p. 24] puts it very well: "instead of identity having the status of an initial given (with which the communication usually begins), it becomes a stake, a product of the 
communication". Status is gained or lost directly from message exchanges, mediated by language.

With practice a great deal can be determined about a poster and, consciously or not, a reader makes value judgements based on message appearance, style and content. Each message will reflect an individual's way of handling language, giving a skilled reader many clues about such status markers as class and education. While it is possible to mislead and lie in e-discourse, it is much harder to alter one's style and general language skills. By watching how discussion and challenges are handled over a series of messages, even more information can be gleaned about an individual's cognitive style. Also, formal layout of the message, use of the subject line, message openings and handling of quotes can all convey information beyond actual content.

\section{Rule governed behaviour}

As with other discursive modes, e-discourse is rule governed behaviour. Just as there are shared assumptions necessary to successful face to face conversation, there are conventions in e-discourse. These range from formal, rules rigidly applied on some premoderated lists, to a mutual assent between those participating in an interaction amidst the anarchy of many FidoNet conferences. Rules vary depending on the setting, whether BBS conference, newsgroup or scholarly list. Competence in more than one type of e-discourse should be encouraged. Ideally, people in education should be able to participate in a variety of types of e-discourse. They should not assume that competence in one form indicates competence in all, nor that behavioural norms for one setting will apply in another.

\section{IMPORTANT ASPECTS}

\section{Understanding the rules of the game}

A greater understanding of e-discourse should be of interest to those tasked with implementing and encouraging the use of related communications technology. Students and other new users need to learn how e-discourse differs from other forms in which they are competent, and why familiar techniques which work on paper or in conversation often will not translate. Since e-discourse is probably another instance of what Lyotard (cited by Feenberg) calls the "atomization of society into flexible networks of language games", [2, p. 26] it may help to explain the rules of the game. As a game, ediscourse has a social content and purpose which may go beyond an e-text's semantic content. Communication, and hence the social relationship it makes possible, can become an end in and of itself. There should be an explicit 
understanding of how a user can win, what the object of the game is, what risks are entailed and what form winning can take.

The game of e-discourse can frequently have a result in which all participants are winners. This helps explain its great attraction and its power. Formal Games Theory invites comparison of e-discourse to the Prisoner's Dilemma, in which cooperation with others, aside from any moral considerations, is the strategy most likely to lead to individual success. However, we are already seeing signs that the early days of electronic altruism in e-discourse are waning, especially on the Internet.

\section{Developing competence in e-discourse}

Competence in e-discourse does not come easily. Exclusively scholarly or work related training is not enough by itself. Learners need to play at these 'flexible networks of language games'. Many competent in e-discourse have as reinforcement an interest in the recreational use of computers and telecommunications. Appreciation for 'tools of the trade' should be encouraged, even enabled by the provision of environments for play wherever possible. To help users identify and assimilate different appropriate behaviours for various electronic environments they will encounter, learners should be exposed to a wide range of e-discourse including e-mail, chats and conferencing, both professional and recreational.

In a recent article for Time about the nature of e-discourse and its quality of writing, Philip Elmer-Dewitt asks "why so much of it is so awful?" [9, p. 52]. A more instructive question is "why is so much of it so good?" The fact remains that in an era when the lack of basic literacy among us is a major social problem, e-discourse, by nature based in text, represents a renewal of both reading and writing.

For educators there is a genuine payoff. Boshier cited Levinson's experience in the mid 1980s at the New York City New School for Social Research [4, p. 53] that:

“... students are sharpening their literary skills. Since our primary mode of communication is writing rather than talking, we have noticed an improvement in the students' writing abilities".

Even the best training will be attenuated if learners receive no immediate gain. There must be an apparent purpose beyond mandatory course requirements. Ideally the enrichment would be felt as a personal or even collective benefit. In his analysis of CMC education, Feenberg [2, p. 29] found that:

"System administrators try to get people on-line with the hope that once they connect something will happen. This approach to CMC leads to 
disappointing results. It ignores the most profound potential of the medium, which is to provide electronically for groups to achieve a common purpose."

\section{RECOMMENDATIONS}

In our experiences, physiotherapy students used an e-mail and conference system, though only sporadically, since they were together in an academic block seeing each other regularly in class. When half were placed in remote communities for clinical experience, use increased dramatically, though only for those students without peers in their community. They were using e-discourse to enhance lateral relationships, geographically separated, for both professional enhancement and peer socialization.

We found that it is not enough to orient students to the medium and expect significant learning and skill development in the time available for one course, unless this is the dominant aspect of the course. It is unreasonable to expect students immersed in regular course content to learn how to cope with a new medium. In such cases they usually learn the minimum required, rarely going beyond the frustration stage. This neither produces competence nor encourages additional independent exploration. A coordinated effort in programmes to produce students competent in e-discourse is needed. Success requires that competence in e-discourse be an explicit goal, not just for students, but also for those who work in education, whether faculty, staff or administrators.

\section{REFERENCES}

1. Harasim, L. M. (1993) Networlds: Networks as social space in Global Networks. Computers and International Communication. (ed. Harasim, L.M.), MIT Press, Cambridge, MA, pp. 15-34.

2. Feenberg, A. (1989) The written world: On the theory and practice of computer conferencing. in Mindweave. Communication, Computers and Distance Education. (eds Mason, R. and Kaye, A.), Pergamon Press, Oxford, pp. 22-39. 
3. Kiesler, S., Siegel, J., and McGuire, T.W. (1991) Social psychological aspects of computer-mediated communication in Computerization and Controversy. Value Conflicts and Social Choices. (eds Dunlop, C. and Kling, R.), Academic Press Inc., Boston, MA, pp. 30-349. [Originally published in American Psychologist (1984), 39 (10) pp. 1123-1134].

4. Boshier, R. (1990) Socio-psychological factors in electronic networking. International Journal of Lifelong Education, 9 (1) pp. 49-64.

5. Van Gelder, L. (1991) The strange case of the electronic lover, in Computerization and Controversy. Value Conflicts and Social Choices. (eds Dunlop, C. and Kling, R.), Academic Press Inc., Boston, MA, pp. 64-75. [Originally published in Ms. Magazine, (1985, October)]

7. Kiesler, S. (1992) Talking, teaching, \& learning in network groups: Lessons from research in Collaborative Learning through Computer Conferencing. The Najaden Papers. (ed Kaye, A. R.), Springer-Verlag, London, pp. 147-165.

8. Grint, K. (1992) Sniffers, lurkers, actor networkers: Computer mediated communications as a technical fix in Technological Literacy and the Curriculum, (eds Beynon, J. and Mackay, H.), Falmer Press, London, pp. 4870.

9. Elmer-Dewitt, P. (1994, July 4) Bards of the Internet. Time (Canadian edition), pp. 52-53. 